\title{
Long non-coding RNAs XIST and MALAT1 hijack the PD-L1 regulatory signaling pathway in breast cancer subtypes
}

\author{
AMANY SAMIR $^{1}$, REDA ABDEL TAWAB ${ }^{2}$ and HEND M. EL TAYEBI ${ }^{1}$ \\ ${ }^{1}$ Molecular Pharmacology Research Group, Department of Pharmacology and Toxicology, German University in Cairo, \\ Cairo 11835; ${ }^{2}$ Department of General Surgery, Ain Shams University, Cairo 11772, Egypt
}

Received July 18, 2020; Accepted January 14, 2021

DOI: $10.3892 / 01.2021 .12854$

\begin{abstract}
Long non-coding RNAs (lncRNAs) have attracted widespread attention as potential biological and pathological regulators. IncRNAs are involved in several biological processes in cancer. Triple negative breast cancer (TNBC) is characterized by strong heterogeneity and aggressiveness. At present, the implication of microRNAs (miRs) and lncRNAs in immunotherapy has been poorly studied. Nevertheless, the blockade of immune checkpoints, particularly that of the programmed cell-death protein-1/programmed cell-death ligand-1 (PD-L1) axis, is considered as a principle approach in breast cancer (BC) therapy. The present study aimed to investigate the interaction between immune-modulatory upstream signaling pathways of the PD-L1 transcript that could enhance personalized targeted therapy. MDA-MB-231 cells were transfected with miR-182-5p mimics followed by RNA extraction and cDNA synthesis using a reverse transcription kit, and the expression levels of the target genes were assessed by reverse transcription-quantitative PCR. Furthermore, the expression levels of target genes were measured in tissues derived from 41 patients with $\mathrm{BC}$, including patients with luminal $\mathrm{BC}$ and TNBC, as well as their adjacent lymph nodes. The results revealed that the expression levels of miR-182-5p, PD-L1 and metastasis-associated lung adenocarcinoma transcript 1 (MALAT1) were upregulated in MDA-MB-231 cells and BC tissues. However, $\mathrm{X}$-inactive specific transcript (XIST) expression was downregulated in cancer tissues and TNBC cells. Following co-transfection of cells with small interfering RNAs specific for each target gene and miR-182-5p antagomirs, the effect of miR-182-5p was abolished in the
\end{abstract}

Correspondence to: $\mathrm{Dr}$ Hend M. El Tayebi, Molecular Pharmacology Research Group, Department of Pharmacology and Toxicology, German University in Cairo, 3rd Settlement, B7, Cairo 11835, Egypt

E-mail: hend.saber@guc.edu.eg

Key words: triple negative breast cancer, immunotherapy, long non-coding RNAs, programmed cell-death ligand-1, metastasis-associated lung adenocarcinoma transcript $1, \mathrm{X}$-inactive specific transcript presence of lncRNAs. Therefore, the results of the present study indicated that although miR-182-5p exhibited an oncogenic effect, XIST exerted a dominant effect on the regulation of the PD-L1 signaling pathway via the inhibition of the oncogenic function of MALAT1.

\section{Introduction}

In the context of tumor biology, the six hallmarks of cancer have been proposed to be associated with progressively growing tumors and to be responsible for the complexity of neoplastic diseases, and these are limitless replicative potential, evading apoptosis, self-sufficiency in growth signals, sustained angiogenesis, insensitivity to anti-growth signals, invasion and metastasis (1). In the last decade, two emerging hallmarks have been added to this list, namely deregulating cellular energetics and evading immune destruction (2). Evasion of immune destruction, resulting in the formation of the tumor microenvironment through a theory known as 'cancer immune-editing', remains a major concern (2). This theory comprises three distinct phases: Elimination, equilibrium and escape (2). Tumor cells induce the immune system, and, in turn, tumor-infiltrating lymphocytes migrate to the tumor site to eradicate the transformed cells (3). However, some of these transformed cells can escape the immune destruction and can progressively grow and give rise to a clinically apparent tumor (3). Therefore, the immune system is considered as a dual weapon; it either suppresses tumor formation or facilitates tumor progression by sculpting the immunogenicity of the tumors (4). This has brought immunotherapy to the forefront of oncology, aiming to inhibit tumor growth and activate antitumor activity. Among the different approaches of immunotherapy, immune checkpoints serve an important role. Notably, programmed cell-death protein-1 (PD-1) is expressed by T-lymphocytic cells (T-cells) during the effector phase to limit its function via binding to its ligand, known as programmed cell-death ligand-1 (PD-L1) on the surface of tumor cells, thus leading to T-cell exhaustion (5). Nivolumab and pembrolizumab are two anti-PD-1 immunotherapies that have been approved for the treatment of melanoma and non-small cell lung cancer (NSCLC), respectively $(6,7)$. Alternative oncogenic signaling pathways promote PD-L1 expression in tumor cells, which is the 'innate immune response'. The induction of PD-L1 expression in response to 
IFN- $\gamma$ is known as the 'adaptive immune response' (8). Based on these important signaling pathways, the regulation of PD-L1 expression is a broad area of investigation in several types of cancer, including breast cancer (BC).

In 2018, BC was the most common type of cancer among women worldwide and ranked first among Egyptian women (9). BC is associated with a poor prognosis due to the strong heterogeneity of its pathogenesis. Disease complexity has prompted researchers to investigate what is beyond the genetic disruption of the disease. The results of these studies revealed that the epigenetic regulation of the disease pathogenesis and progression also serves an important role in BC. Emerging evidence has suggested that the newly discovered non-coding RNAs (ncRNAs) greatly contribute to carcinogenesis (10). microRNAs (miRNAs/miRs), a subtype of ncRNAs, may lead to gene silencing via binding to the $3^{\prime}$ untranslated region (3'UTR) of target mRNAs, either through translational repression or mRNA cleavage (11). Several miRNAs, such as Let-7a and miR-145, have been reported to be tumor suppressors in BC, resulting in decreased cellular proliferation and metastasis (12). Another class of miRNAs that contribute to cancer cell proliferation are oncomiRs, such as miR-10b and miR-21 (13). The present study focused on miR-182-5p, which has been reported to serve as either an oncogene or tumor suppressor in numerous types of cancer. Previous studies have demonstrated that the inhibition of miR-182-5p attenuates cell proliferation and invasion in BC (14), hepatocellular carcinoma (HCC) (15) and oral squamous cell carcinoma (16). Furthermore, miR-182-5p suppresses renal cell carcinoma cell proliferation by regulating the AKT/FOXO3a signaling pathway (17).

Another important group of ncRNAs are long non-coding RNAs (lncRNAs). IncRNAs serve a pivotal role in gene silencing and disease progression (18). The IncRNA X-inactive specific transcript (XIST) is exclusively expressed from the $\mathrm{X}$-inactivation center of the inactive $\mathrm{X}$ chromosome and is essential for the initiation and spread of $\mathrm{X}$ chromosome inactivation (19). A previous review article reported that XIST exerts contradictory functions in different types of cancer (20). For example, in invasive pituitary adenoma, XIST acts as an oncogene (21). In addition, XIST could promote brain metastasis following its silencing in BC (22). Our previous study demonstrated that XIST combined with PD-L1 expression could serve as a potential biomarker in patients with BC (23). Furthermore, a recent study has supported the role of PD-L1 as a useful biomarker for immunotherapy (24). Another study revealed that PD-L1 expression is positively associated with that of lncRNA T cell leukemia/lymphoma 6 (TCL6) (25). In addition, it has been reported that IncRNA TCL6 is associated with a poor prognosis in patients with $\mathrm{BC}$ and increased immune cell infiltration (25). Additionally, lncRNA GATA binding protein 3 antisense RNA 1 induces the deubiquitination of PD-L1, thus resulting in PD-L1 stabilization and enhanced triple-negative breast cancer (TNBC) progression (26). TSIX transcript, XIST antisense RNA (TSIX) is considered to orchestrate the initiation of $\mathrm{X}$ chromosome inactivation, thus determining which $\mathrm{X}$ chromosome remains active by blocking the expression of the antisense XIST RNA (27). Another lncRNA, metastasis-associated lung adenocarcinoma transcript 1 (MALAT1), was originally identified as a prognostic marker for metastatic lung cancer (28); however, it is also associated with several other human tumors, such as HCC (29) and glioblastoma (30). Therefore, the current study aimed to identify potential ncRNAs regulating PD-L1 expression in TNBC cell lines.

\section{Materials and methods}

Egyptian patients. The present study included 41 patients with BC (4.88\% males and $95.12 \%$ females) who underwent tumor resection surgery between September 2016 and April 2018 at the following hospitals: Demerdash, Cleopatra, Queens and Nozha Hospitals (Cairo, Egypt). BC tissues biopsies as well as their adjacent non-cancerous tissues together with their metastatic lymph nodes (LNs) were removed. Tissues were subdivided into luminal $\mathrm{BC}(\mathrm{n}=30 ; 73.1 \%)$, TNBC $(\mathrm{n}=7$; $17.07 \%)$ and HER-2-positive $(\mathrm{n}=4 ; 9.75 \%)$ subtype. The age of patients ranged between 28 and 70 years with a mean of 49 years. Pathological examination was performed to assess tumor grade and stage (The Eighth Edition of the American Joint Committee Cancer Staging Manual) using the TNM staging system (31). Immunohistochemistry was performed to analyze receptors (estrogen receptor, progesterone receptor and HER2) and Ki67. Tumor molecular subtyping was performed for all tumor tissues by a pathologist during the surgical resection, it was not performed at our laboratory. This was performed at Elia Laboratory (Cleopatra Hospital, Heliopolis, Cairo, Egypt). Furthermore, CEA and CA15-3 were analyzed before surgery. All human biopsies were obtained with written informed consent. Patients were subjected to clinical assessment as shown in Table I. The Ethical Committee of the German University in Cairo and Ain Shams University (Cairo, Egypt) approved the present study. The inclusion criteria were: All molecular subtypes of $\mathrm{BC}$, all ages and all types of treatment. The exclusion criteria were: Male sex.

Cell culture. MDA-MB-231 cells (Vacsera) were cultured and maintained in DMEM (Lonza Group, Ltd.) supplemented with $4.5 \mathrm{~g} / 1$ glucose + L-Glutamine, 10\% FBS (Lonza Group, Ltd.) and $1 \%$ penicillin/streptomycin (Lonza Group, Ltd.) at $37^{\circ} \mathrm{C}$ in a $5 \% \mathrm{CO}_{2}$ atmosphere.

Bioinformatics. To detect the potential miRNAs targeting the 3'UTR of PD-L1 mRNA, the TargetScan (release number, 7.2; http://www.targetscan.org/vert_72/) bioinformatics target prediction algorithm was used. Based on binding scores and number of hits, miRNAs with good scores were selected. PD-L1 upstream targets were predicted. RNA22 software version 2.0 (http://cm.jefferson.edu/rna22/Interactive/) competing endogenous RNA (ceRNA; version 2.0; https://web.archive.org/web/20130922123437/http://starbase.sysu. edu.cn/mrnaCeRNA.php) and TargetScan prediction software were used to analyze the potential binding of miR-182-5p to lncRNAs XIST, MALAT1 and PD-L1 (position 1193-1199 in the UTR). Furthermore, 1 Cedb (Gencode 19 version; http://gyanxet-beta.com/lncedb/index.php) and Diana tools software (version 7.0; http://diana.imis.athena-innovation. $\mathrm{gr} /$ DianaTools/index.php? $\mathrm{r}=$ site/page \& view=software) were used to predict the potential binding of lncRNAs XIST and MALAT1 to PD-L1. 
Table I. Characteristics of patients with breast cancer.

\begin{tabular}{|c|c|c|c|c|c|c|c|c|c|}
\hline No. & Size, $\mathrm{cm}$ & Type & Grade & Stage & $\begin{array}{c}\text { Axillary } \\
\text { lymph node }\end{array}$ & Treatment & $\begin{array}{c}\text { Duration of } \\
\text { cancer since diagnosis }\end{array}$ & $\begin{array}{l}\text { Molecular } \\
\text { subtype }\end{array}$ & $\mathrm{Ki} 67, \%$ \\
\hline 1 & 4.0 & IDC & 3 & 4 & Positive & N/A & 2 months & TNBC & 35 \\
\hline 2 & 2.0 & IDC & 3 & 1 & Positive & N/A & 2 months & Luminal B, HER2- & 40 \\
\hline 3 & 2.4 & IDC & 2 & 2 & Positive & N/A & 6 months & Luminal B, HER2+ & 40 \\
\hline 4 & 4.0 & IDC & 3 & 2 & Positive & N/A & 6 months & Luminal B, HER2- & 15 \\
\hline 5 & 2.5 & IDC & 2 & 3 & Negative & $\begin{array}{l}\text { Neoadjuvant } \\
\text { chemotherapy } \\
\text { (6 cycles) }\end{array}$ & 8 months & Luminal B, HER $2^{+}$ & 25 \\
\hline 6 & 2.5 & IDC & 2 & 2 & Positive & N/A & 2 months & Luminal B, HER2 & 23 \\
\hline 7 & 1.4 & IDC & 2 & 1 & Negative & N/A & 2 months & Luminal A & 12 \\
\hline 8 & 2.1 & IDC & 2 & 2 & Negative & N/A & 4 months & Luminal B, HER2- & 23 \\
\hline 9 & 1.4 & IDC & 2 & 1 & Negative & N/A & 3 months & Luminal B, HER2 & 30 \\
\hline 10 & 3.5 & IDC & 2 & 2 & Negative & N/A & 1 year & Luminal A & 17 \\
\hline 11 & 5.0 & IDC & 3 & 3 & Negative & $\mathrm{N} / \mathrm{A}$ & 6 months & $\mathrm{HER}^{+}$ & 50 \\
\hline 12 & 0.3 & IDC & 2 & 2 & Negative & $\begin{array}{c}\text { Chemotherapy and } \\
\text { radiotherapy }\end{array}$ & 1 month & TNBC & 30 \\
\hline 13 & 2.0 & IDC & 2 & 2 & Negative & N/A & 1 month & TNBC & 35 \\
\hline 14 & 2.7 & IDC & 2 & 2 & Positive & N/A & 2 weeks & Luminal B, HER $2^{+}$ & 30 \\
\hline 15 & 4.2 & IDC & 2 & 2 & Negative & N/A & 6 months & $\mathrm{HER}^{+}{ }^{+}$ & 40 \\
\hline 16 & 2.0 & IDC & 2 & 2 & Negative & $\mathrm{N} / \mathrm{A}$ & 2 months & Luminal B, HER 2+ & 14 \\
\hline 17 & 2.0 & IDC & 2 & 2 & Negative & $\begin{array}{l}\text { Chemotherapy and } \\
\text { radiotherapy }\end{array}$ & 1 month & Luminal A & 18 \\
\hline 18 & 4.0 & IDC & 2 & 2 & Positive & $\mathrm{N} / \mathrm{A}$ & 8 months & Luminal B & 24 \\
\hline 19 & 2.5 & IDC & 2 & 2 & Negative & N/A & 3 months & Luminal B, HER2- & 50 \\
\hline 20 & 3.0 & IDC & 2 & 2 & Positive & N/A & 4 months & TNBC & 85 \\
\hline 21 & 0.3 & IDC & 2 & 1 & Positive & N/A & 1 month & HER $2+^{+}$ & 30 \\
\hline 22 & 3.0 & IDC & 2 & 2 & Positive & N/A & 4 months & Luminal A & 20 \\
\hline 23 & 4.0 & ILC & 2 & 2 & Positive & N/A & 6 months & Luminal A & 18 \\
\hline 24 & 1.5 & IDC & 1 & 1 & Negative & N/A & 2 years & Luminal A & 14 \\
\hline 25 & $6.0 \times 3.0$ & IDC & 2 & 2 & Positive & N/A & 6 months & Luminal A & 5 \\
\hline 26 & $2.0 \times 2.5$ & IDC & 3 & 3 & Positive & N/A & 10 months & TNBC & 18 \\
\hline 27 & 2.5 & IDC & 2 & 2 & Negative & N/A & 2 years & Luminal A & 10 \\
\hline 28 & $2.5 \times 2.3$ & IDC & 2 & 2 & Positive & N/A & 7 months & Luminal B & 35 \\
\hline 29 & $2.5 \times 2.0$ & IDC & 2 & 3 & Positive & N/A & 8 months & Luminal B & 22 \\
\hline 30 & 4.0 & IDC & 3 & 4 & Negative & N/A & 6 months & Luminal B & 60 \\
\hline 31 & $1.0 \times 1.0$ & IDC & 3 & N/A & Positive & N/A & 6 months & Luminal B, HER2- & 35 \\
\hline 32 & 9.0 & IDC & 2 & N/A & Negative & N/A & 1 year & Luminal B & 50 \\
\hline 33 & 4.2 & IDC & 2 & 2 & Positive & N/A & 1 month & Luminal B & 30 \\
\hline 34 & 1.6 & ILC & 1 & 2 & Negative & N/A & 2 months & Luminal B & 22 \\
\hline 35 & $2.5 \times 2.0$ & IDC & 2 & 2 & Positive & N/A & 6 months & $\mathrm{HER}^{+}{ }^{+}$ & 35 \\
\hline 36 & $1.5 \times 1.0$ & IDC & 2 & 1 & Positive & N/A & 4 months & Luminal A & 8 \\
\hline 37 & $3.5 \times 2.5$ & IDC & 2 & 2 & Positive & N/A & 3 months & TNBC & 30 \\
\hline 38 & 1.8 & IDC & 2 & 1 & Negative & N/A & 1 month & Luminal A & 7 \\
\hline 39 & $2.0 \times 1.5$ & IDC & 2 & 2 & Positive & N/A & 4 months & TNBC & 30 \\
\hline 40 & $2.5 \times 2.0$ & IDC & 2 & 2 & Positive & N/A & 2 months & Luminal A & 18 \\
\hline 41 & $4.0 \times 3.0$ & IDC & 2 & 4 & Positive & N/A & 6 months & Luminal A & 15 \\
\hline
\end{tabular}

IDC, invasive ductal carcinoma; TNBC, triple-negative breast cancer.

Transfection of MDA-MB-231 cells using miRNA and small interfering RNA (siRNA/si) oligonucleotides. MDA-MB-231 cells were transfected with $1 \mathrm{nmol}$ mimics (GeneGlobe, cat. no. 219600) and inhibitors (antagomiRs) of $1 \mathrm{nmol}$ 
miR-182-5p (GeneGlobe Id-MIN0000259; 5'-UUUGGCAAU GGUAGAACUCACACU-3'; cat. no. 219300; Qiagen GmbH) at $25^{\circ} \mathrm{C}$ for $1 \mathrm{~h}$ to examine the effect of miR-182-5p on PD-L1, MALAT1, XIST and TSIX transcript expression. In addition, a parallel experiment was carried out for transfection efficiency analysis. This was followed by a series of transfection experiments using 5 nmol siRNAs (predesigned siRNA; Qiagen $\mathrm{GmbH}$ ) for each IncRNA, MALAT1 (NR_002819), XIST (NR_001564) and TSIX (NR_003255). Co-transfection experiments were performed to examine the combined effect of the upstream manipulators (miR-182-5p and lncRNAs XIST and MALAT1) of PD-L1 on its expression levels. All transfection experiments were carried out in quadruplicate using HiPerfect Transfection Reagent (Qiagen $\mathrm{GmbH}$ ) according to the manufacturer's protocol. A group of scrambled (non-specific) siRNAs (cat. no. 1022076; Qiagen $\mathrm{GmbH}$ ) and scrambled miRNA mimics and antagomirs: Mixtures of mimics of miR-15a-5p (cat. no. 219600) and miR-122 (cat. no. 219600) for scrambled miRs and mixtures of anti-miR-15a-5p and anti-miR-122 for scrambled anti-miRs (hsa-miR-15a-5p; MIMAT0000068; 5'-UAGCAGCACAUAAUGGUUUGUG-3'; cat. no. 219300; and hsa-miR-122-5p; MIMAT0000421; 5'-UGGAGUGUG ACAAUGGUGUUUG-3'; cat. no. 219300; Qiagen GmbH) were used as negative controls in gene knockdown and miRNA gain/loss of function experiments, respectively. Cells that were only exposed to transfection reagent were designated as mock cells, cells transfected with miR-182-5p were referred to as miR-182-5p cells and cells transfected with miR-182-5p inhibitor were referred to as anti-miR-182-5p cells. Cells transfected with siRNAs of MALAT1, XIST and TSIX were referred to as siMALAT1, siXIST and siTSIX, respectively. The cells were transfected and incubated under normal culture conditions $\left(37^{\circ} \mathrm{C}\right.$ with $\left.5 \% \mathrm{CO}_{2}\right)$ for $48 \mathrm{~h}$.

mRNA and miRNA extraction from breast biopsies and MDA-MB-231 cells (TNBC cell lines). Breast samples (healthy, cancerous and adjacent $\mathrm{LN}$ tissues) were collected during surgery and were immediately snap-frozen $\left(-196^{\circ} \mathrm{C}\right)$ in liquid nitrogen. The specimens were manually pulverized in liquid nitrogen. Subsequently, $100 \mathrm{mg}$ tissue powder was used for large and small RNA extraction using Biozol reagent (BioFlux) according to the manufacturer's protocol. MDA-MB-231 cells were harvested $48 \mathrm{~h}$ after transfection according to the HiPerfect Transfection Reagent protocol. RNA was isolated using Biozol reagent, followed by cDNA synthesis using a High-Capacity cDNA Reverse Transcription Kit (Applied Biosystems; Thermo Fisher Scientific, Inc.) at $37^{\circ} \mathrm{C}$ for $135 \mathrm{~min}$. Subsequently, RNA was quantified using reverse transcription-quantitative PCR (RT-qPCR). Experiments were performed in quadruplicate.

miRNA and mRNA quantification. The extracted miRNAs were reverse transcribed into single stranded cDNA using the TaqMan MicroRNA Reverse Transcription Kit (Bio Basic, Inc.). mRNA was reverse transcribed into cDNA using the high-capacity cDNA reverse transcription kit (Bio Basic, Inc.) according to the manufacturer's protocol at $37^{\circ} \mathrm{C}$ for 75 min. Relative expression levels of miR-182-5p and RNU6B (housekeeping gene) were measured using specific primers for hsa-miR-182-5p and RNU6B. Their assay IDs were 002334 and 001093, respectively, as well as MALAT1, XIST, PD-L1, TSIX and $\beta-2$ microglobulin (as a housekeeping gene for normalization) were quantified using TaqMan Real-Time Q-PCR (Applied Biosystems; Thermo Fisher Scientific, Inc.). The ABI Assay IDs for MALAT1, XIST, PD-L1, TSIX and B2M were Hs00273907_m1, Hs01079824_m1, Hs01125301_ m1, Hs03299334_ml and Hs00187842_m1, respectively. A StepOne $^{\mathrm{TM}}$ System (Applied Biosystems; Thermo Fisher Scientific, Inc.) was used. The $2^{-\Delta \Delta \mathrm{Cq}}$ method was used for quantification (32). The thermocycling conditions were as follows: $25^{\circ} \mathrm{C}$ for $10 \mathrm{~min}, 37^{\circ} \mathrm{C}$ for $120 \mathrm{~min}, 85^{\circ} \mathrm{C}$ for $5 \mathrm{~min}, 4^{\circ} \mathrm{C}$ for infinity consisting of 40 cycles of denaturation, annealing and extension, respectively.

Statistical analysis. All data are presented as the mean relative quantitation \pm SEM and repeated in quadruplicates. The statistical method used for multiple groups was one-way ANOVA and multiple comparisons were analyzed by Tukey's multiple comparison test (when the mean of each column was compared with every other column) and Dunnett's multiple comparison test (when the mean of each column was compared with the mean of the control column). Analysis was performed using GraphPad Prism 7.02 software (GraphPad Software, Inc.). $\mathrm{P}<0.05$ was considered to indicate a statistically significant difference.

\section{Results}

Screening of PD-L1 in breast tissues. Statistically significant upregulation of $\mathrm{PD}-\mathrm{L} 1$ transcript expression was observed in all $\mathrm{BC}$ subtype tissues $(\mathrm{P}=0.0217)$ compared with healthy tissues, whereas this change was not significant in the LNs compared with adjacent normal tissues ( $\mathrm{P}=0.0360$; Fig. 1A). However, when patients were categorized into luminal molecular subtypes and TNBC, a marked difference in PD-L1 expression was observed. Patients with TNBC exhibited significant upregulation of $\mathrm{PD}-\mathrm{L} 1$ expression $(\mathrm{P}=0.0037)$ compared with patients with luminal subtypes (Fig. 1B).

Selection of potential upstream regulators of PD-L1 mRNA. In silico predictions were performed using all aforementioned software. According to bioinformatics analysis, miR-182-5p was predicted to target PD-L1, MALAT1 and XIST. Additionally, MALAT1 and XIST were identified to target PD-L1 mRNA.

Screening of miR-182-5p, MALAT1 and XIST expression in $B C$ tissues. miR-182-5p expression was identified to be upregulated in tumor tissues and LNs $(\mathrm{P}=0.0061$ and $\mathrm{P}=0.0014$, respectively) compared with in healthy tissues (ANOVA $\mathrm{P}=0.0013$; Fig. 2A). A marked increase in MALAT1 mRNA expression was observed in patients with all subtypes of $\mathrm{BC}$ compared with normal adjacent tissue controls $(\mathrm{P}=0.00001)$, whereas there was no significant difference observed for the expression in LNs (ANOVA P $<0.0001$; Fig. 2B). In our previous study, XIST expression was decreased in tissues of patients with BC and adjacent $\mathrm{LN}$ samples from these patients, and markedly downregulated in TNBC (23).

Transfection efficiency for gene knockdown and miRNA ectopic expression. In order to assure successful transfection 

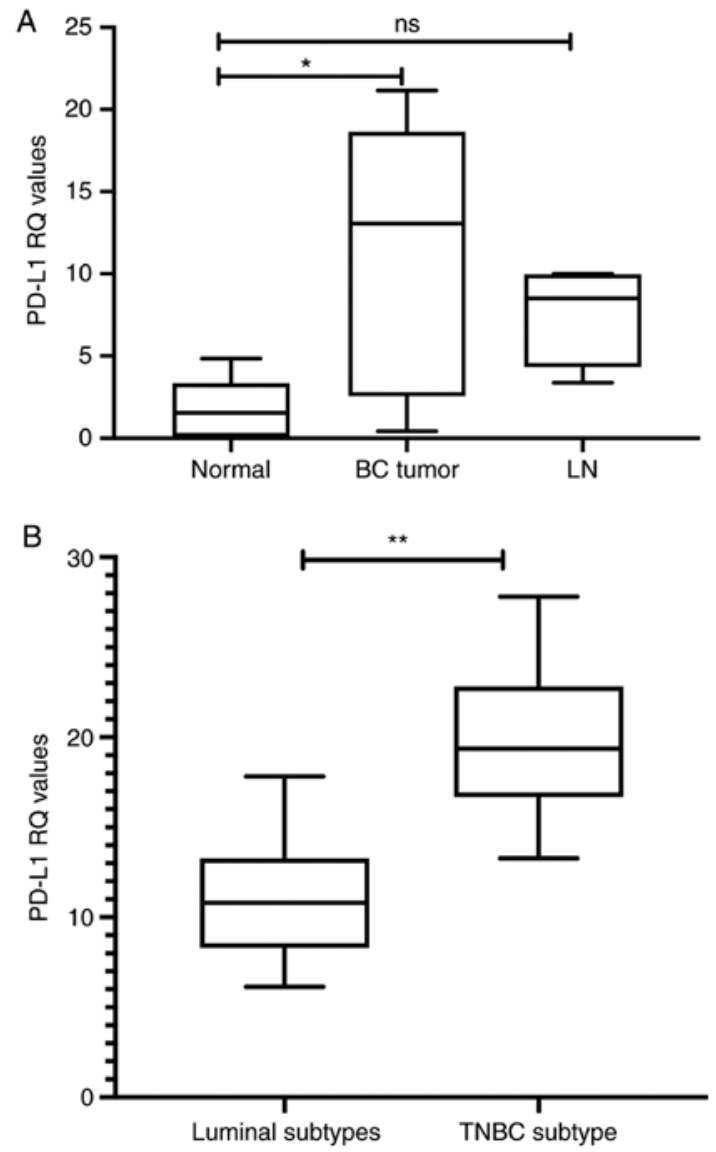

Figure 1. PD-L1 expression in breast tissues. (A) PD-L1 expression was revealed to be significantly upregulated in tumor tissues compared with adjacent normal tissues and non-significant in LNs. Analysis was performed using one-way ANOVA for multiple groups with Dunnett's multiple comparison test. (B) Following further separation of TNBC from other luminal subtypes, PD-L1 expression was identified to be significantly higher in TNBC samples compared with luminal subtypes. CD274 is the cluster of differentiation for PD-L1. Analysis was performed using GraphPad Prism 7.02 software. The expression levels were compared using an unpaired Student's t-test. " $\mathrm{P}<0.05$, ${ }^{* *} \mathrm{P}<0.01$. Experiments were performed in quadruplicate. $\mathrm{BC}$, breast cancer; LN, lymph node; ns, not significant; PD-L1, programmed cell-death ligand-1; $\mathrm{RQ}$, relative quantitation; TNBC, triple-negative breast cancer.

of siRNAs, transfection efficiency was first assessed at $48 \mathrm{~h}$ after transfection using RT-qPCR. The mRNA expression levels of MALAT1 (Fig. S1A), XIST (Fig. S1B) and Tsix (Fig. S1C) were markedly decreased in cells transfected with their siRNAs compared with their respective mock cells $(\mathrm{P}=0.0021, \mathrm{P}=0.0056$ and $\mathrm{P}=0.0051$, respectively). Additionally, the expression levels of miR-182-5p were assessed in MDA-MB-231 cells. miR-182-5p expression was markedly increased in miR-182-transfected cells compared with mock cells ( $\mathrm{P}=0.0044$; Fig. S1D).

Effect of ectopic miR-182-5p expression on its downstream targets in MDA-MB-231 cells. Ectopic miR-182-5p expression in MDA-MB-231 cells was assessed. MDA-MB-231 cells transfected with miR-182-5p mimics exhibited significant upregulation of PD-L1 expression, as well as MALAT1 transript expression $(\mathrm{P}=0.0062$ and $\mathrm{P}=0.0007$, respectively), compared with mock untransfected cells (ANOVA $\mathrm{P}=0.0065$ and 0.0004 , respectively; Fig. $3 \mathrm{~A}$ and $\mathrm{B}$ ). Inhibitors of miR-182-5p significantly decreased PD-L1 and MALAT1
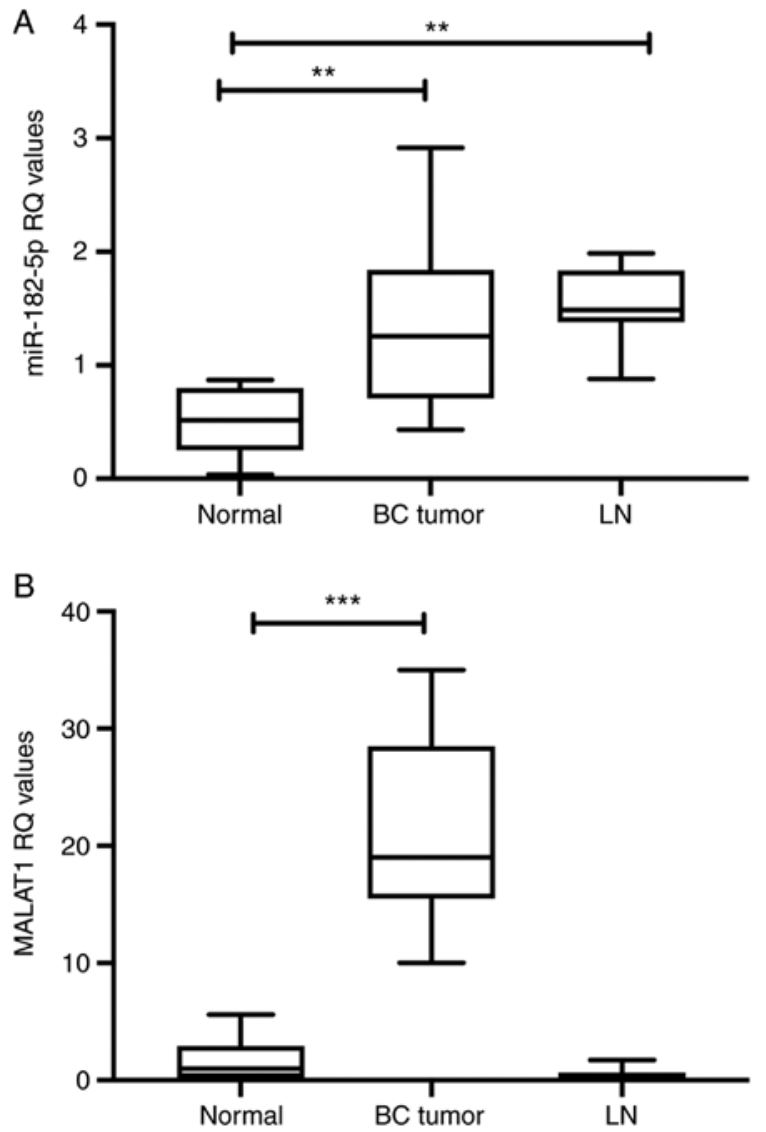

Figure 2. miR-182-5p, MALAT1 expression in BC tissues. (A) miR-182-5p was analyzed using RT-qPCR and normalized to RNU6B as an endogenous control. BC samples, as well as LN samples, exhibited upregulation of miR-182-5p expression. Analysis was performed using one-way ANOVA for multiple groups and Tukey's multiple comparison test. (B) lncRNA MALAT1 expression was analyzed using RT-qPCR and normalized to $\mathrm{B} 2 \mathrm{M}$ as an endogenous control. MALAT1 expression was upregulated in $\mathrm{BC}$ tissues compared with healthy tissues, whereas no significant difference in expression was observed in LNs. Analysis was performed using one-way ANOVA for multiple groups and Dunnett's multiple comparison test. Experiments were performed in quadruplicate. ${ }^{* *} \mathrm{P}<0.01,{ }^{* * *} \mathrm{P}<0.001$. BC, breast cancer; LN, lymph node; lncRNA, long non-coding RNA; MALAT1, metastasis-associated lung adenocarcinoma transcript 1; miR, microRNA; RQ, relative quantitation; RT-qPCR, reverse transcription-quantitative PCR; XIST, X-inactive specific transcript.

transcript expression ( $\mathrm{P}=0.034$ and $\mathrm{P}=0.0053$, respectively) compared with miR-182-transfected cells (Fig. 3A and B). However, transfection of mimics of miR-182-5p was associated with a significant decrease in XIST expression $(\mathrm{P}=0.0026)$ compared with that in the scrambled miRs group of cells, while miR-182-5p antagomirs increased XIST expression compared with that in cells transfected with mimics ( $\mathrm{P}=0.0434$; ANOVA $\mathrm{P}=0.0001$; Fig. 3C). Since Tsix is the anti-sense of XIST, the expression levels of IncRNA Tsix were examined. In MDA-MB-231 cells, overexpression of miR-182-5p increased Tsix expression compared with that in mock cells $(\mathrm{P}=0.0004$; ANOVA P<0.0001; Fig. 3D).

Effect of IncRNAs MALAT1 and XIST on PD-L1 expression in MDA-MB-231 cells. At $48 \mathrm{~h}$ after transfection with specific siRNAs against MALAT1 and XIST, PD-L1 expression was analyzed and normalized to that of B2M.PD-L1 mRNA expression was decreased significantly in MALAT1-silenced MDA-MB-231 

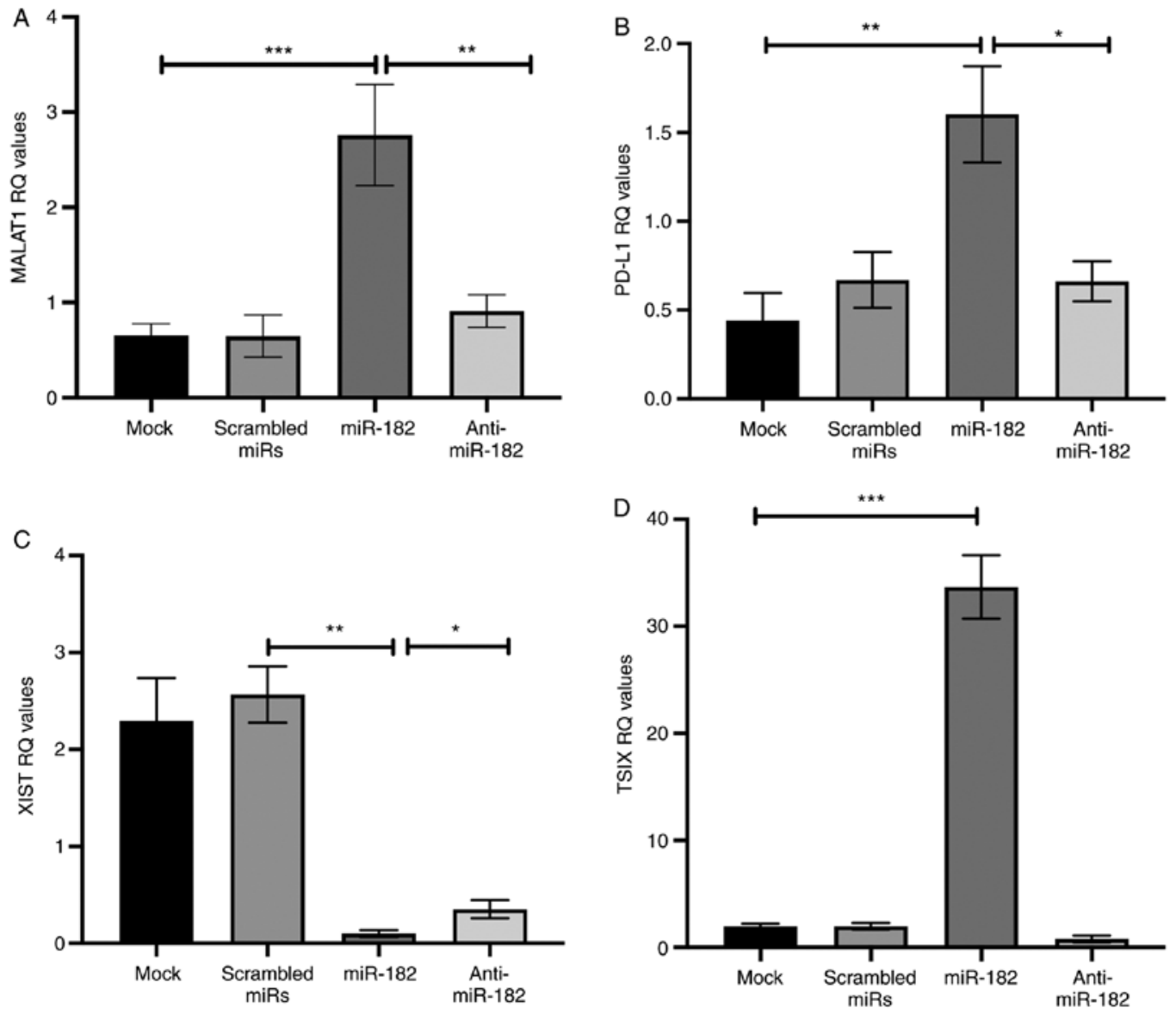

Figure 3. Effect of ectopic miR-182-5p expression on its downstream targets in MDA-MB-231 cells. (A) miR-182-5p mimic transfection of MDA-MB-231 cells resulted in significant upregulation of MALAT1 mRNA expression compared with that in mock cells, whereas anti-miR-182-5p transfection decreased MALAT1 expression compared with mimic-transfected cell lines. (B) PD-L1 mRNA expression was assessed using reverse transcription-quantitative PCR and normalized to B2M as an endogenous control. miR-182-5p mimic transfection of MDA-MB-231 cells resulted in significant upregulation of PD-L1 mRNA expression compared with that in mock cells, whereas anti-miR-182-5p transfection decreased PD-L1 expression compared with that in miR-182-transfected cells. (C) XIST mRNA expression was decreased significantly in miR-182-transfected cells compared with scrambled miRs. Furthermore, anti-miR-182-5p transfection resulted in an increase in XIST expression compared with that in mimic-transfected cells. (D) miR-182-5p mimic transfection resulted in significant upregulation of TSIX expression compared with that in mock cells. Analysis was performed using one-way ANOVA for multiple groups and Tukey's multiple comparison test. Experiments were performed in quadruplicate. ${ }^{*} \mathrm{P}<0.05,{ }^{* * *} \mathrm{P}<0.01,{ }^{* * *} \mathrm{P}<0.001$. MALAT1, metastasis-associated lung adenocarcinoma transcript 1 ; miR, microRNA; PD-L1, programmed cell-death ligand-1; RQ, relative quantitation; XIST, X-inactive specific transcript; Tsix, TSIX transcript, XIST antisense RNA.

cells $(\mathrm{P}=0.0007)$ compared with mock cells (ANOVA $\mathrm{P}=0.0004$; Fig. 4A). In contrast to overexpression of PD-L1, in XIST-silenced MDA-MB-231 cells, PD-L1 mRNA expression was increased significantly in $\mathrm{BC}$ cells $(\mathrm{P}=0.0178)$ compared with untransfected mock cells (ANOVA $\mathrm{P}=0.0071 ;$ Fig. $4 \mathrm{~B}$ ).

Combined effect of the ncRNAs on PD-L1 expression in MDA-MB-231 cells. In three groups of MDA-MB-231 cells, co-transfection of miR-182-5p mimics was performed once with siXIST and PD-L1 mRNA expression was significantly increased compared with that of mock cells $(\mathrm{P}=0.00004)$. In order to induce XIST expression in BC cells, another group of cells was transfected with siRNAs of Tsix, a negative regulator of XIST, combined with mimics of miR-182-5p. miR-182-siTsix co-transfection resulted in a significant decrease in PD-L1 mRNA expression in BC cells, compared with that in mock cells $(\mathrm{P}=0.0357)$. Additionally, the third group of cells was co-transfected with miR-182-5p mimics combined with siMALAT1, and the expression levels of PD-L1 were significantly decreased compared with those in mock cells ( $\mathrm{P}=0.0331$; ANOVA $\mathrm{P}=0.0003$; Fig. 5).
Effect of combined knockdown of lncRNAs on PD-L1 expression in MDA-MB-231 cells. PD-L1 expression was analyzed at $48 \mathrm{~h}$ after transfection of MDA-MB-231 cells. Following transfection with MALAT1 siRNAs combined with XIST siRNAs, a marked decrease in PD-L1 expression was observed compared with that of mock cells $(\mathrm{P}=0.006)$. Furthermore, following silencing of MALAT1 combined with Tsix knockdown, significant downregulation of PD-L1 mRNA expression was observed compared with mock cells $(\mathrm{P}=0.0002$; ANOVA $\mathrm{P}=0.0004$; Fig. 6).

Effect of miR-182-5p mimic transfection combined with lncRNAs on MALAT1 expression in MDA-MB-231 cells. MALAT1 expression was evaluated at $48 \mathrm{~h}$ after transfection of MDA-MB-231 cells with miR-182-5p mimics combined with XIST siRNAs. Based on the results of RT-qPCR, transfection with mimics of miR-182-5p and XIST siRNAs resulted in a significant increase in MALAT1 expression compared with that of mock cells $(\mathrm{P}=0.0004)$. Additionally, combined knockdown of Tsix and ectopic miR-182-5p expression was associated with significant upregulation of MALAT1 

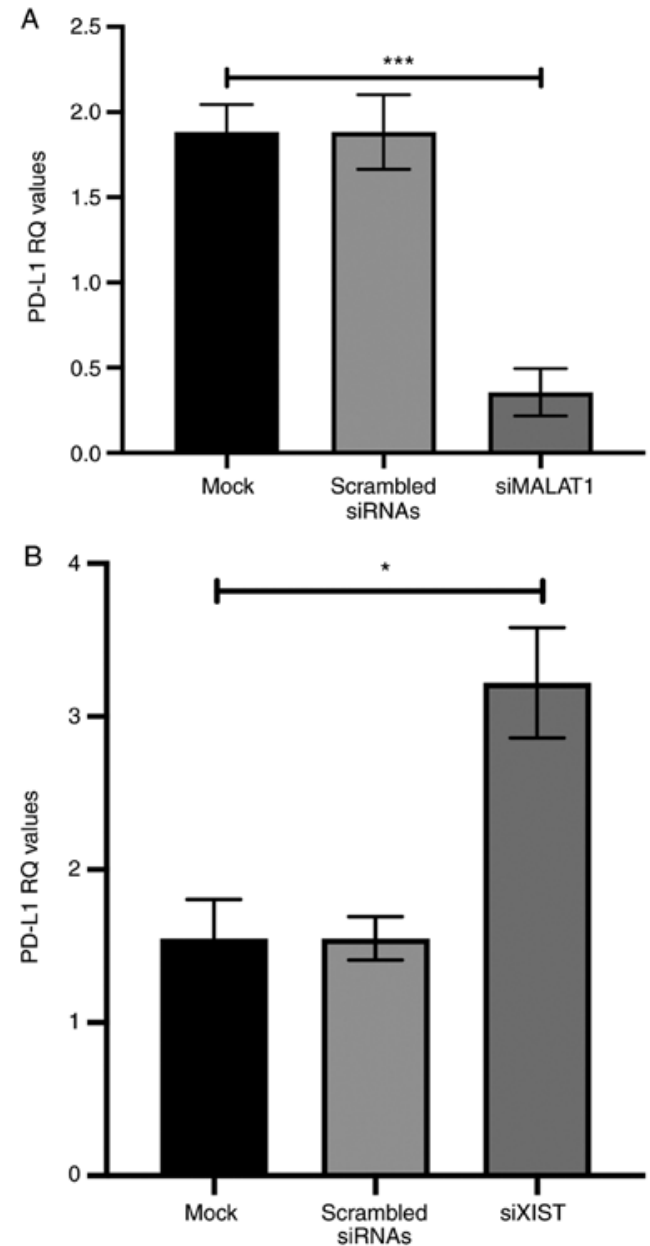

Figure 4. Effects of lncRNAs MALAT1 and XIST on PD-L1 expression in MDA-MB-231 cells. (A) MALAT1 knockdown decreased PD-L1 expression compared with that in mock cells. (B) XIST knockdown increased PD-L1 mRNA expression significantly compared with that in mock cells. Analysis was performed using one-way ANOVA for multiple groups and Dunnett's multiple comparison test. Experiments were performed in quadruplicate. ${ }^{*} \mathrm{P}<0.05,{ }^{* * *} \mathrm{P}<0.001$. IncRNA, long non-coding RNA; MALAT1, metastasis-associated lung adenocarcinoma transcript 1; PD-L1, programmed cell-death ligand-1; RQ, relative quantitation; si, small interfering RNA; XIST, X-inactive specific transcript.

expression compared with that of mock cells $(\mathrm{P}<0.0001$; ANOVA P<0.0001; Fig. 7).

\section{Discussion}

In the last decade, significant progress and previous advances in cancer immunology have provided novel therapeutic approaches for the treatment of cancer (33). The clinical response observed in patients treated with antibodies blocking the immune checkpoints, namely the expression of cytotoxic T-lymphocyte-associated protein 4 and the PD-1/PD-L1 signaling pathway, led to their approval by the Food and Drug Administration for the treatment of melanoma in 2011 and 2014, respectively (34). The antibody against PD-1, nivolumab, was approved in 2015 for squamous lung cancer treatment (6). In addition, it has been reported that antibodies targeting PD-1 or PD-L1 are effective and safe in treating several types of tumors, including bladder cancer, Hodgkin's lymphoma and renal cell carcinoma (35). The effects of immune checkpoint inhibitors in

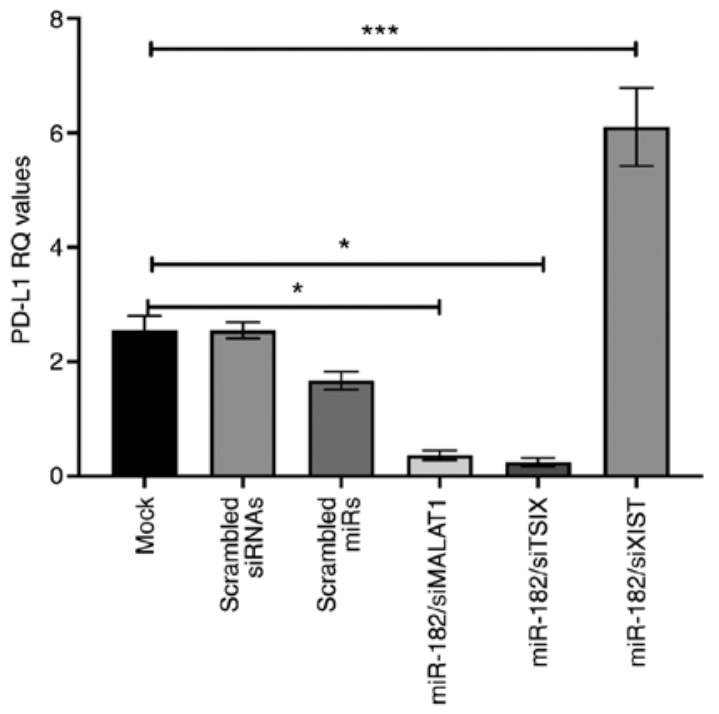

Figure 5. Combined effect of the two upstream regulators of the PD-L1 promoter (miR-182-5p and lncRNAs; XIST and MALAT1) in MDA-MB-231 cells. Following transfection of miR-182-5p mimics and silencing of XIST, PD-L1 expression was increased compared with that of mock cells. However, following transfection with miR-182-5p mimic and knockdown of Tsix, PD-L1 expression was identified to be significantly downregulated, and PD-L1 expression was revealed to be downregulated following transfection with miR-182-5p mimic and silencing of MALAT1. Analysis was performed using one-way ANOVA for multiple groups and Dunnett's multiple comparison test. Experiments were performed in quadruplicate. ${ }^{*} \mathrm{P}<0.05,{ }^{* * * *} \mathrm{P}<0.001$. MALAT1, metastasis-associated lung adenocarcinoma transcript 1 ; miR, microRNA; PD-L1, programmed cell-death ligand-1; RQ, relative quantitation; si, small interfering RNA; XIST, $\mathrm{X}$-inactive specific transcript; Tsix, TSIX transcript, XIST antisense RNA.

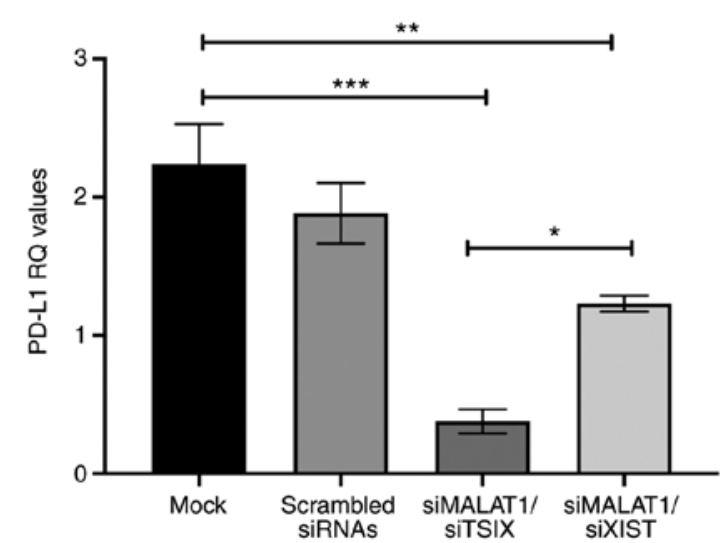

Figure 6. Effect of combined knockdown of lncRNAs on PD-L1 expression in MDA-MB-231 cells. The change in PD-L1 expression as a response to co-transfection of the cells using siRNAs of MALAT1 and XIST was assessed using TaqMan reverse transcription-quantitative PCR and normalized to B2M. The results revealed a marked decrease in PD-L1 expression compared with that in mock cells, in addition to a significant decrease in PD-L1 expression following silencing of the oncogenic MALAT1 and silencing of Tsix, the antisense of XIST. Analysis was performed using one-way ANOVA for multiple groups and Tukey's multiple comparison test. Experiments were performed in quadruplicate. ${ }^{*} \mathrm{P}<0.05,{ }^{* *} \mathrm{P}<0.01,{ }^{* * *} \mathrm{P}<0.001$. lncRNA, long on-coding RNA; MALAT1, metastasis-associated lung adenocarcinoma transcript 1; PD-L1, programmed cell-death ligand-1; RQ, relative quantitation; si, small interfering RNA; XIST, X-inactive specific transcript; Tsix, TSIX transcript, XIST antisense RNA.

different types of cancer have promoted the targeting of these signaling pathways in other tumors, such as BC (36). The association between PD-L1 and the prognosis of 


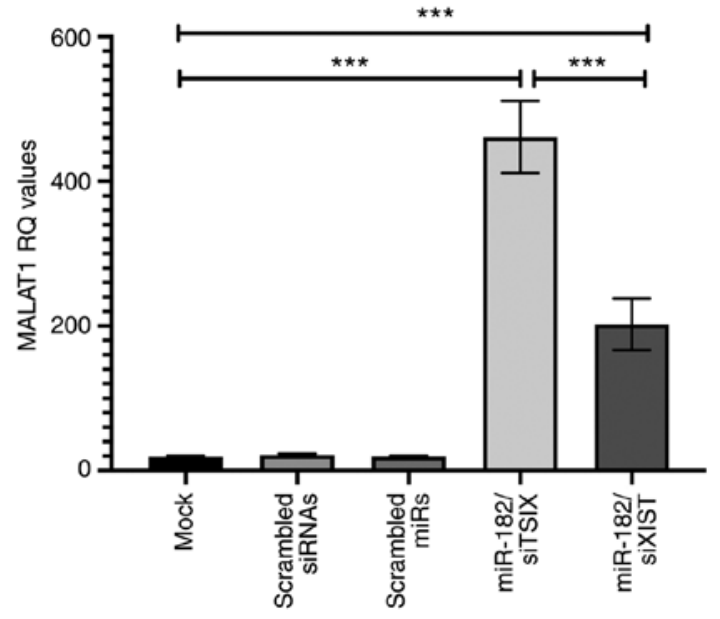

Figure 7. Effect of miR-182-5p mimic transfection combined with lncRNAs on MALAT1 regulation in MDA-MB-231 cells. Using reverse transcription-quantitative PCR, the relative expression levels of MALAT1 were analyzed and normalized to B2M. A significant increase in MALAT1 expression was observed in breast cancer cells when XIST siRNAs were co-transfected with miR-182-5p mimics compared with mock cells. Notably, MALAT1 mRNA expression was increased when breast cancer cells were co-transfected with siTsix combined with miR-182-5p mimics compared with that of mock cell. Analysis was performed using one-way ANOVA for multiple groups and Tukey's multiple comparison test. Experiments were performed in quadruplicate ${ }^{* * *} \mathrm{P}<0.001$. IncRNA, long on-coding RNA MALAT1, metastasis-associated lung adenocarcinoma transcript 1; miR, microRNA; RQ, relative quantitation; si, small interfering RNA; XIST, $\mathrm{X}$-inactive specific transcript; Tsix, TSIX transcript, XIST antisense RNA.

patients with different types of cancer has been a research topic of considerable interest. However, the prognostic value of PD-L1 in patients with BC remains controversial. Therefore, the current study aimed to investigate the differential expression of lncRNAs in tissues derived from patients with BC and the MDA-MB-231 cell line. In addition, the miRNA-mediated regulation of PD-L1, XIST and MALAT1 expression remains poorly investigated, particularly in BC. Therefore, the present study also aimed to reveal novel miRNA/lncRNA interactions in BC and their immune-modulatory effects on PD-L1 expression, in order to provide novel possible immunotherapeutic targets for the treatment of different subtypes of $\mathrm{BC}$, particularly TNBC. The results demonstrated that PD-L1 expression was upregulated in $\mathrm{BC}$ tissues, with higher expression levels observed in TNBC compared with the luminal subtypes. A previous study has demonstrated that PD-L1 expression is associated with LN metastasis and TNBC, suggesting that PD-L1 could serve as a promising biomarker for monitoring prognosis in patients with $\mathrm{BC}$ and selecting the appropriate immunotherapy (37). Consistent with a previous study that demonstrated that PD-L1 expression is upregulated in the MDA-MB-468 cell line (38), the PD-L1 expression levels were increased in MDA-MB-231 cells in the present study. Early clinical trials have revealed that treatment with PD-1/PD-L1 inhibitors is efficient against BC tumors, and particularly against TNBC (39). Furthermore, a previous study has demonstrated that patients with metastatic TNBC present a positive clinical response to the blockade of PD-1 or PD-L1 with specific antibodies, such as pembrolizumab or atezulizumab (40). This finding was consistent with the results of the present study. Notably, PD-L1 expression is associated with several clinicopathological parameters, including tumor size, grade and invasion (41). PD-L1 expression is associated with higher tumor grade (42), shortened survival rate and poor therapeutic outcome $(43,44)$.

Since the present study demonstrated that PD-L1 expression was upregulated in TNBC, bioinformatics tools were subsequently applied to identify the upstream modulators of its expression. It has been reported that ncRNAs are involved in $\mathrm{BC}$ and the regulation of gene expression. The majority of studies have focused on determining the functions of miRNAs and lncRNAs, and only a few have investigated how their expression is transcriptionally regulated. Furthermore, numerous studies have reported the targeting effect of miRNAs on lncRNAs in different types of cancer. For example, a study demonstrated that miR-130a could directly target FOS-like antigen 1, thus inhibiting cancer cell migration and invasion in TNBC (45). In addition, lncRNAs can compete with miRNAs for the same target-gene and they serve as precursors for miRNAs. Emerging evidence has suggested that miRNAs are critical key players in cancer immunotherapy as they act as crucial regulators of immune responses under physiological and pathological conditions (46). It has been demonstrated that miRNAs are involved in cell transformation and multiplication by acting as oncomiRs or tumor suppressors in various types of cancer (47). Several miRNAs have been identified as regulators of PD-L1 expression. Tumor suppressors, such as miR-15a and miR-16, are predicted to target PD-L1, thus resulting in downregulation of PD-L1 expression in malignant pleural mesothelioma (48). In the present study, miR-182-5p was selected based on the results of the bioinformatics analysis, and it was demonstrated that miR-182-5p exerted strong binding affinity with PD-L1, MALAT1 and XIST mRNAs. The results revealed that miR-182-5p expression was upregulated in TNBC, as well as in luminal subtypes. This finding was consistent with previous studies, showing that miR-182-5p expression is increased in TNBC $(49,50)$. Additionally, another study revealed that treatment with miR-182-5p inhibitors attenuates cell apoptosis and proliferation via regulation of CRISPR associated protein 9 expression in MCF-7 human BC cells (51). Additionally, miR-182-5p has been identified to be upregulated in TNBC and luminal A breast tumors (52). Furthermore, miR-182-5p was highly expressed in a panel of human BC samples, highlighting its role as a potential oncomiR in $\mathrm{BC}$ that could positively regulate metastasis and promote cell colonization (53).

lncRNA MALAT1 serves an important oncogenic role in different types of cancer. The present study demonstrated that MALAT1 mRNA expression was significantly elevated in $\mathrm{BC}$ tissues. These findings were in agreement with previous studies showing that lncRNA MALAT1 could promote cell proliferation and invasion in TNBC (54) and lung cancer (55). Additionally, miR-182-5p could regulate MALAT1 expression in MDA-MB-231 cells. A previous study revealed that MALAT1 overexpression is associated with poor prognosis in patients with colorectal carcinoma (53). In addition, the expression levels of MALAT1 are positively associated with the LN status, tumor stage and histological grade in BC (54). 
MALAT1 downregulation suppresses the progression of osteosarcoma (56). Furthermore, miR-129-5p could upregulate MALAT1, resulting in cell proliferation and the progression of colon cancer (57). Previous studies have demonstrated that MALAT1 serves a protumorigenic role in pancreatic cancer (58), NSCLC (59) and ovarian cancer (60). Furthermore, accumulating evidence has suggested that MALAT1 contributes to the initiation and progression of bladder cancer via regulation of the expression levels of miRNAs (61). A previous study suggested that MALAT1 could serve as a therapeutic target or a novel diagnostic biomarker for BC (62). Consistent with the findings of the present study, another study demonstrated that MALAT1 knockdown inhibits $\mathrm{BC}$ cell proliferation, migration and invasion, and induces apoptosis (63). The present results revealed that silencing of MALAT1 decreased PD-L1 expression. Furthermore, it has been demonstrated that lysine demethylase $5 \mathrm{~B}$ expression could promote BC aggressiveness via MALAT1 overexpression and downregulation of miR-448 (64). In TNBC, MALAT1 expression is upregulated, and patients with increased levels of MALAT1 exhibit poor overall survival (65). It has been reported that IncRNA XIST serves an important role as a tumor suppressor or oncogene in several types of cancer, such as prostate cancer where it acts as a tumor suppressor (66). In the present study, XIST expression was downregulated in MDA-MB-231 cells following miR-182-5p overexpression. Similarly, a previous study revealed that XIST expression is downregulated in BC (67). By contrast, another study demonstrated that the expression levels of XIST were increased in BRCA1-positive BC, suggesting that XIST expression could be used as a marker to discriminate between BRCA1-positive and -negative breast tumors (68). Additionally, XIST upregulation promotes osteosarcoma (69), HCC (70) and bladder cancer (71) cell proliferation, while it acts as an oncogene in NSCLC via regulation of the miR-37a/la-related protein 1 downstream signaling pathway (72).

Since PD-L1 and MALAT1 act as immune-modulatory targets in the scope of the downstream signaling pathway, their expression pattern was investigated following manipulation of miR-182-5p expression. Ectopic miR-182-5p expression was assessed in MDA-MB-231 cells using RT-qPCR and resulted in the upregulation of PD-L1 and TSIX expression. In addition, elevated mRNA expression levels of MALAT1 were observed in MDA-MB-231 cells transfected with miR-182-5p mimics compared with mock cells. In contrast to renal cancer, where miR-182-5p mimics decrease MALAT1 expression, resulting in inhibition of cancer cell proliferation (73), the treatment with miR-182-5p inhibitor reversed this effect. Transfection with miR-182-5p mimics markedly downregulated XIST expression. Bioinformatics analysis predicted that PD-L1 could strongly bind with MALAT1 and XIST. Therefore, it was hypothesized that the regulation of PD-L1 expression could be mediated by lncRNAs, as upstream regulators, rather than miR-182-5p. Therefore, the effect of each lncRNA on PD-L1 expression was investigated. The expression levels of PD-L1 were determined in cells transfected with siMALAT1 or siXIST. PD-L1 expression was upregulated following transfection with siXIST; however, it was downregulated following MALAT1 knockdown. This finding was consistent with the results of another study, demonstrating that the expression levels of MALAT1 are positively associated with PD-L1 expression in NSCLC (59). Additionally, following treatment of B-cell lymphoma human cell lines with short hairpin RNA MALAT1, PD-L1 levels are decreased, resulting in inhibition of tumor cell proliferation (74).

To investigate the regulatory association between miR-182-5p and PD-L1, and to explore the combined effect of the two upstream factors on the regulation of PD-L1 expression, the expression levels of PD-L1 were assessed in cells co-transfected with different modulators. PD-L1 expression was downregulated in cells co-transfected with siMALAT1 and miR-182-5p mimics. Furthermore, silencing of Tsix (a negative regulator of XIST) and miR-182-5p overexpression in MDA-MB-231 cells decreased PD-L1 expression. However, the mRNA expression levels of PD-L1 were increased in MDA-MB-231 cells following co-transfection with siXIST and miR-182-5p mimics. Additionally, it was hypothesized that the effect of miR-182-5p on PD-L1 expression was abolished in the presence of lncRNAs. Therefore, the combined effect of IncRNA expression on that of PD-L1 was further investigated. MDA-MB-231 cells were co-transfected with siMALAT1 and siXIST or siMALAT1 combined with siTsix to upregulate XIST expression. For both co-transfection conditions, PD-L1 mRNA expression levels were evaluated. The results revealed that following the silencing of both MALAT1 and Tsix, PD-L1 expression was downregulated compared with that of control cells. In addition, the expression levels of PD-L1 were decreased in MDA-MB-231 cells transfected with siXIST and siMALAT1. These opposing forces on the regulation of PD-L1 expression indicated that XIST could augment the inhibitory effect of MALAT1 knockdown on PD-L1 expression. This hypothesis prompted an investigation into the effect of the main lncRNAs, MALAT1 and XIST, on PD-L1 expression. Therefore, the expression levels of MALAT1 were determined in cells co-transfected with miR-182-5p mimics and siXIST. The results revealed that MALAT1 expression was upregulated in the aforementioned cells. Additionally, MALAT1 expression was upregulated in cells co-transfected with miR-182-5p mimics and siTsix. Notably, PD-L1 expression was downregulated in these cells, suggesting that XIST could be the dominant endogenous competitor in the regulation of PD-L1 expression. Nevertheless, the lack of experiments in additional TNBC cell lines should be considered to be a potential limitation of the present study.

In conclusion, the present study introduced a novel immune-modulatory miRNA-lncRNA interaction network in BC, namely the MALAT1/XIST/miR-182-5p/PD-L1 axis. A previous study (75) has demonstrated that miR-182 acts as an oncomiR, since its expression increases cell migration and proliferation in vitro. In vivo assays in mice have demonstrated that the expression of miR-182 significantly increases tumor volume and enhances instant metastasis in the lungs (75). The results of the present study suggested that the upregulation of miR-182-5p could act as an oncomiR in BC tissues and MDA-MB-231 cells, and highlighted its molecular effects on pivotal immunomodulatory signaling pathways by promoting the upregulation of oncogenic lncRNAs PD-L1 and MALAT1 in the MDA-MB-231 BC cell line. In addition, miR-182-5p 
downregulated the expression of the tumor suppressor gene XIST in the same cells. These findings supported the key role of the ceRNA network, MALAT1/XIST, in regulating $\mathrm{PD}-\mathrm{L} 1$ expression in $\mathrm{BC}$, and suggested their potential role as immunotherapeutic targets. Overall, both molecules could be utilized as promising biomarkers in clinical diagnosis and prognosis of aggressive BC tumors.

\section{Acknowledgements}

Not applicable.

\section{Funding}

No funding was received.

\section{Availability of data and materials}

The datasets used and/or analyzed during the current study are available from the corresponding author on reasonable request.

\section{Authors' contributions}

AS performed the practical work, data analysis, writing and revision of the manuscript. RAT was the sample provider. RAT was also involved in the acquisition of data, editing and revising the manuscript, and contributed to the conception and design of the study, and to obtaining materials and analysis tools, as well as assessing the authenticity of all the raw data. HMET was the principle investigator of the project who supervised the work. HMET made substantial contributions to conception and design, in addition to analysis and interpretation of data, was involved in drafting the manuscript, assessing the authenticity of all the raw data and revising it critically for important intellectual content and gave final approval of the version to be published. All authors read and approved the final manuscript.

\section{Ethics approval and consent to participate}

Written informed consent for participation in the study or use of their tissue was obtained from all participants. The Ethical Committee of the German University in Cairo and Ain Shams University (Cairo, Egypt) approved the present study.

\section{Patient consent for publication}

Not applicable.

\section{Competing interests}

The authors declare that they have no competing interests.

\section{References}

1. Fouad YA and Aanei C: Revisiting the hallmarks of cancer. Am J Cancer Res 7: 1016-1036, 2017.

2. Mittal D, Gubin MM, Schreiber RD and Smyth MJ: New insights into cancer immunoediting and its three component phases-elimination, equilibrium and escape. Curr Opin Immunol 27: 16-25, 2014.
3. Vaziri Fard E, Ali Y, Wang XI, Saluja K, H Covinsky M, Wang L and Zhang S: Tumor-infiltrating lymphocyte volume is a better predictor of disease-free survival than stromal tumor-infiltrating lymphocytes in invasive breast carcinoma. Am J Clin Pathol 152: 656-665, 2019.

4. Chen Y, Wang J, Wang X, Li X, Song J, Fang J, Liu X, Liu T, Wang D, Li Q, et al: Pik3ip1 is a negative immune regulator that inhibits antitumor T cell immunity. Clin Cancer Res 25: 6180-6194, 2019.

5. Alsaab HO, Sau S, Alzhrani R, Tatiparti K, Bhise K, Kashaw SK and Iyer AK: PD-1 and PD-L1 checkpoint signaling inhibition for cancer immunotherapy: Mechanism, combinations, and clinical outcome. Front Pharmacol 8: 561, 2017.

6. Guo L, Zhang H and Chen B: Nivolumab as Programmed Death-1 (PD-1) inhibitor for targeted immunotherapy in tumor. J Cancer 8: 410-416, 2017.

7. McDermott $\mathbf{J}$ and Jimeno A: Pembrolizumab: PD-1 inhibition as a therapeutic strategy in cancer. Drugs Today (Barc) 51: 7-20, 2015.

8. Karachaliou N, Gonzalez-Cao M, Crespo G, Drozdowskyj A, Aldeguer E, Gimenez-Capitan A, Teixido C, Molina-Vila MA, Viteri S, De Los Llanos Gil M, et al: Interferon gamma, an important marker of response to immune checkpoint blockade in non-small cell lung cancer and melanoma patients. Ther Adv Med Oncol: Jan 18, 2018 (Epub ahead of print). doi: $10.1177 / 1758834017749748$.

9. Azim HA and Ibrahim AS: Breast cancer in Egypt, China and Chinese: Statistics and beyond. J Thorac Dis 6: 864-866, 2014.

10. Prensner JR and Chinnaiyan AM: The emergence of lncRNAs in cancer biology. Cancer Discov 1: 391-407, 2011.

11. Jin HY and Xiao C: MicroRNA mechanisms of action: What have we learned from mice? Front Genet 6: 328, 2015.

12. Sempere LF, Christensen M, Silahtaroglu A, Bak M, Heath CV, Schwartz G, Wells W, Kauppinen S and Cole CN: Altered MicroRNA expression confined to specific epithelial cell subpopulations in breast cancer. Cancer Res 67: 11612-11620, 2007.

13. Asghari F, Haghnavaz N, Baradaran B, Hemmatzadeh M and Kazemi T: Tumor suppressor microRNAs: Targeted molecules and signaling pathways in breast cancer. Biomed Pharmacother 81: 305-317, 2016.

14. Zhao YS, Yang WC, Xin HW, Han JX and Ma SG: MiR-182-5p knockdown targeting PTEN inhibits cell proliferation and invasion of breast cancer cells. Yonsei Med J 60: 148-157, 2019.

15. Cao MQ, You AB, Zhu XD, Zhang W, Zhang YY, Zhang SZ, Zhang KW, Cai H, Shi WK, Li XL, et al: miR-182-5p promotes hepatocellular carcinoma progression by repressing FOXO3a. J Hematol Oncol 11: 12, 2018.

16. Li N, Nan CC, Zhong XY, Weng JQ, Fan HD, Sun HP, Tang S, Shi L and Huang SX: miR-182-5p promotes growth in oral squamous cell carcinoma by inhibiting CAMK2N1. Cell Physiol Biochem 49: 1329-1341, 2018.

17. Xu X, Wu J, Li S, Hu Z, Xu X, Zhu Y, Liang Z, Wang X, Lin Y, Mao Y, et al: Downregulation of microRNA-182-5p contributes to renal cell carcinoma proliferation via activating the AKT/FOXO3a signaling pathway. Mol Cancer 13: 109, 2014.

18. Kopp F and Mendell JT: Functional classification and experimental dissection of long noncoding RNAs. Cell 172: 393-407, 2018.

19. Fang H, Disteche CM and Berletch JB: X Inactivation and escape: Epigenetic and structural features. Front Cell Dev Biol 7: 219,2019

20. Samir A, Salama E and El Tayebi HM: The long non-coding RNA XIST: A new cornerstone in carcinogenesis. J Mol Genet Med 12: 2, 2018.

21. Zhou K, Li S, Du G, Fan Y, Wu P, Sun H and Zhang T: LncRNA XIST depletion prevents cancer progression in invasive pituitary neuroendocrine tumor by inhibiting bFGF via upregulation of microRNA-424-5p. Onco Targets Ther 12: 7095-7109, 2019.

22. Xing F, Liu Y, Wu SY, Wu K, Sharma S, Mo YY, Feng J, Sanders S, Jin G, Singh R, et al: Loss of XIST in breast cancer activates MSN-c-Met and reprograms microglia via exosomal miRNA to promote brain metastasis. Cancer Res 78: 4316-4330, 2018.

23. Salama EA, Adbeltawab RE and El Tayebi HM: XIST and TSIX: Novel cancer immune biomarkers in PD-L1-overexpressing breast cancer patients. Front Oncol 9: 1459, 2019.

24. Yu Y, Zhang W, Li A, Chen Y, Ou Q, He Z, Zhang Y, Liu R, Yao H and Song E: Association of long noncoding RNA biomarkers with clinical immune subtype and prediction of immunotherapy response in patients with cancer. JAMA Netw Open 3: e202149, 2020 . 
25. Zhang Y, Li Z, Chen M, Chen H, Zhong Q, Liang L and Li B: lncRNA TCL6 correlates with immune cell infiltration and indicates worse survival in breast cancer. Breast Cancer 27: 573-585, 2020.

26. Zhang M, Wang N, Song P, Fu Y, Ren Y, Li Z and Wang J: LncRNA GATA3-AS1 facilitates tumour progression and immune escape in triple-negative breast cancer through destabilization of GATA3 but stabilization of PD-L1. Cell Prolif 53 e12855, 2020.

27. Gayen S, Maclary E, Buttigieg E, Hinten M and Kalantry S: A primary role for the Tsix lncRNA in maintaining random X-chromosome inactivation. Cell Rep 11: 1251-1265, 2015.

28. Zhao ZB, Chen F and Bai XF: Long noncoding RNA MALAT1 regulates hepatocellular carcinoma growth under hypoxia via sponging MicroRNA-200a. Yonsei Med J 60: 727-734, 2019.

29. Ji P, Diederichs S, Wang W, Böing S, Metzger R, Schneider PM, Tidow N, Brandt B, Buerger H, Bulk E, et al: MALAT-1, a novel noncoding RNA, and thymosin beta4 predict metastasis and survival in early-stage non-small cell lung cancer. Oncogene 22: 8031-8041, 2003

30. Argadal OG, Mutlu M, Ak Aksoy S, Kocaeli H, Tunca B Civan MN, Egeli U, Cecener G, Bekar A, Taskapilioglu MO, et al: Long noncoding RNA MALAT1 may be a prognostic biomarker in IDH1/2 wild-type primary glioblastomas. Bosn J Basic Med Sci 20: 63-69, 2020

31. Amin MB, Edge S, Greene F, Byrd DR, Brookland RK, Washington MK, Gershenwald JE, Compton CC, Hess KR, Sullivan DC, et al (eds). AJCC Cancer Staging Manual. 8th edition. Springer International Publishing, 2017.

32. Livak KJ and Schmittgen TD: Analysis of relative gene expression data using real-time quantitative PCR and the 2(-Delta Delta C(T)) method. Methods 25: 402-408, 2001.

33. Mittendorf EA, Philips AV, Meric-Bernstam F, Qiao N, Wu Y, Harrington S, Su X, Wang Y, Gonzalez-Angulo AM, Akcakanat A, et al: PD-L1 expression in triple-negative breast cancer. Cancer Immunol Res 2: 361-370, 2014.

34. Blackburn SD, Shin H, Haining WN, Zou T, Workman CJ Polley A, Betts MR, Freeman GJ, Vignali DA and Wherry EJ: Coregulation of $\mathrm{CD} 8+\mathrm{T}$ cell exhaustion by multiple inhibitory receptors during chronic viral infection. Nat Immunol 10: 29-37, 2009.

35. Buchbinder EI and Desai A: CTLA-4 and PD-1 pathways: Similarities, differences, and implications of their inhibition. Am J Clin Oncol 39: 98-106, 2016

36. Linsley PS, Brady W, Urnes M, Grosmaire LS, Damle NK and Ledbetter JA: CTLA-4 is a second receptor for the B cell activation antigen B7. J Exp Med 174: 561-569, 1991.

37. van der Merwe PA, Bodian DL, Daenke S, Linsley P and Davis SJ: CD80 (B7-1) binds both CD28 and CTLA-4 with a low affinity and very fast kinetics. J Exp Med 185: 393-403, 1997.

38. Sasidharan Nair V, Toor SM, Ali BR and Elkord E: Dual inhibition of STAT1 and STAT3 activation downregulates expression of PD-L1 in human breast cancer cells. Expert Opin Ther Targets 22: 547-557, 2018.

39. Monneur A,Gonçalves A and Bertucci F: PD-L1 expression and PD-1/PD-L1 inhibitors in breast cancer. Bull Cancer 105 263-274, 2018 (In French).

40. Alegre ML, Frauwirth KA and Thompson CB: T-cell regulation by CD28 and CTLA-4. Nat Rev Immunol 1: 220-228, 2001

41. Wang X, Teng F, Kong L and Yu J. PD-L1 expression in human cancers and its association with clinical outcomes. Onco Targets Ther 9: 5023-5039, 2016.

42. Takahashi T, Tagami T, Yamazaki S, Uede T, Shimizu J, Sakaguchi N, Mak TW and Sakaguchi S: Immunologic self-tolerance maintained by $\mathrm{CD} 25(+) \mathrm{CD} 4(+)$ regulatory $\mathrm{T}$ cells constitutively expressing cytotoxic $\mathrm{T}$ lymphocyte-associated antigen 4. J Exp Med 192: 303-310, 2000.

43. Krummel MF and Allison JP: CTLA-4 engagement inhibits IL-2 accumulation and cell cycle progression upon activation of resting T cells. J Exp Med 183: 2533-2540, 1996

44. Wing K, Onishi Y, Prieto-Martin P, Yamaguchi T, Miyara M, Fehervari Z, Nomura T and Sakaguchi S: CTLA-4 control over Foxp3+ regulatory T cell function. Science 322: 271-275, 2008

45. Mao H, Zhang L, Yang Y, Zuo W, Bi Y, Gao W, Deng B, Sun J, Shao Q and Qu X: New insights of CTLA-4 into its biological function in breast cancer. Curr Cancer Drug Targets 10: 728-736, 2010.

46. Duraiswamy J, Kaluza KM, Freeman GJ and Coukos G: Dual blockade of PD-1 and CTLA-4 combined with tumor vaccine effectively restores T-cell rejection function in tumors. Cancer Res 73: 3591-3603, 2013.
47. Hodi FS, O'Day SJ, McDermott DF, Weber RW, Sosman JA, Haanen JB, Gonzalez R, Robert C, Schadendorf D, Hassel JC, et al: Improved survival with ipilimumab in patients with metastatic melanoma. N Engl J Med 363: 711-723, 2010.

48. Kao SC, Cheng YY, Williams M, Kirschner MB, Madore J, Lum T, Sarun KH, Linton A, McCaughan B, Klebe S, et al: Tumor suppressor microRNAs contribute to the regulation of PD-L1 expression in malignant pleural mesothelioma. J Thorac Oncol 12: 1421-1433, 2017.

49. Schütz F, Stefanovic S, Mayer L, von Au A, Domschke C and Sohn C: PD-1/PD-L1 pathway in breast cancer. Oncol Res Treat 40: 294-297, 2017.

50. Krishnan K, Steptoe AL, Martin HC, Wani S, Nones K, Waddell N, Mariasegaram M, Simpson PT, Lakhani SR, Gabrielli B, et al: MicroRNA-182-5p targets a network of genes involved in DNA repair. RNA 19: 230-242, 2013.

51. Dong H, Strome SE, Salomao DR, Tamura H, Hirano F, Flies DB Roche PC, Lu J, Zhu G, Tamada K, et al: Tumor-associated B7-H1 promotes T-cell apoptosis: A potential mechanism of immune evasion. Nat Med 8: 793-800, 2002.

52. Yee D, Shah KM, Coles MC, Sharp TV and Lagos D: MicroRNA-155 induction via TNF-alpha and IFN-gamma suppresses expression of programmed death ligand-1 (PD-L1) in human primary cells. J Biol Chem 292: 20683-20693, 2017.

53. Zhan Y, Li X, Liang X, Li L, Cao B, Wang B, Ma J, Ding F, Wang X, Pang D and Liu Z: MicroRNA-182 drives colonization and macroscopic metastasis via targeting its suppressor SNAI1 in breast cancer. Oncotarget 8: 4629-4641, 2017.

54. Robainas M, Otano R, Bueno S and Ait-Oudhia S: Understanding the role of PD-L1/PD1 pathway blockade and autophagy in cancer therapy. Onco Targets Ther 10: 1803-1807, 2017.

55. Wu F, Yin Z, Yang L, Fan J, Xu J, Jin Y, Yu J, Zhang D and Yang G: Smoking induced extracellular vesicles release and their distinct properties in non-small cell lung cancer. J Cancer 10: 3435-3443, 2019.

56. Cha YJ and Shim HS: PD-L1 expression and CD8+ tumor-infiltrating lymphocytes are associated with ALK rearrangement and clinicopathological features in inflammatory myofibroblastic tumors. Oncotarget 8: 89465-89474, 2017.

57. Peters S, Kerr KM and Stahel R: PD-1 blockade in advanced NSCLC: A focus on pembrolizumab. Cancer Treat Rev 62 39-49, 2018

58. Li L, Chen H, Gao Y, Wang YW, Zhang GQ, Pan SH, Ji L, Kong R, Wang G, Jia YH, et al: Long noncoding RNA MALAT1 promotes aggressive pancreatic cancer proliferation and metastasis via the stimulation of autophagy. Mol Cancer Ther 15: 2232-2243, 2016

59. Wei S, Wang K, Huang X, Zhao Z and Zhao Z: LncRNA MALAT1 contributes to non-small cell lung cancer progression via modulating miR-200a-3p/programmed death-ligand 1 axis. Int J Immunopathol Pharmacol 33: 2058738419859699, 2019.

60. Gordon MA, Babbs B, Cochrane DR, Bitler BG and Richer JK: The long non-coding RNA MALAT1 promotes ovarian cancer progression by regulating RBFOX2-mediated alternative splicing. Mol Carcinog 58: 196-205, 2019.

61. Xie H, Liao X, Chen Z, Fang Y, He A, Zhong Y, Gao Q, Xiao H, Li J, Huang W and Liu Y: LncRNA MALAT1 inhibits apoptosis and promotes invasion by antagonizing miR-125b in bladder cancer cells. J Cancer 8: 3803-3811, 2017.

62. Ou X, Gao G, Bazhabayi M,Zhang K, Liu Fand Xiao X: MALAT1 and $\mathrm{BACH} 1$ are prognostic biomarkers for triple-negative breast cancer. J Cancer Res Ther 15: 1597-1602, 2019.

63. Xiping Z, Bo C, Shifeng Y, Feijiang Y, Hongjian Y, Qihui C and Binbin T: Roles of MALAT1 in development and migration of triple negative and Her-2 positive breast cancer. Oncotarget 9: 2255-2267, 2018.

64. Bamodu OA, Huang WC, Lee WH, Wu A, Wang LS, Hsiao M, Yeh CT and Chao TY: Aberrant KDM5B expression promotes aggressive breast cancer through MALAT1 overexpression and downregulation of hsa-miR-448. BMC Cancer 16: 160, 2016

65. Zheng L, Zhang Y, Fu Y, Gong H, Guo J, Wu K, Jia Q and Ding X: Long non-coding RNA MALAT1 regulates BLCAP mRNA expression through binding to miR-339-5p and promotes poor prognosis in breast cancer. Biosci Rep 39: BSR20181284, 2019.

66. Du Y, Weng XD, Wang L, Liu XH, Zhu HC, Guo J, Ning JZ and Xiao CC: LncRNA XIST acts as a tumor suppressor in prostate cancer through sponging miR-23a to modulate RKIP expression. Oncotarget 8: 94358-94370, 2017. 
67. Jiang L, Yu X, Ma X, Liu H, Zhou S, Zhou X, Meng Q, Wang L and Jiang W: Identification of transcription factor-miRNA-lncRNA feed-forward loops in breast cancer subtypes. Comput Biol Chem 78: 1-7, 2019.

68. Schouten PC, Vollebergh MA, Opdam M, Jonkers M, Loden M, Wesseling J, Hauptmann M and Linn SC: High XIST and Low 53BP1 expression predict poor outcome after high-dose alkylating chemotherapy in patients with a BRCA1-like breast cancer. Mol Cancer Ther 15: 190-198, 2016.

69. Yang C, Wu K, Wang S and Wei G: Long non-coding RNA XIST promotes osteosarcoma progression by targeting YAP via miR-195-5p. J Cell Biochem 119: 5646-5656, 2018.

70. Liu WG and Xu Q: Long non-coding RNA XIST promotes hepatocellular carcinoma progression by sponging miR-200b-3p. Eur Rev Med Pharmacol Sci 23: 9857-9862, 2019.

71. Zhou K, Yang J, Li X and Chen W: Long non-coding RNA XIST promotes cell proliferation and migration through targeting miR-133a in bladder cancer. Exp Ther Med 18: 3475-3483, 2019.
72. Xu Z, Xu J, Lu H, Lin B, Cai S, Guo J, Zang F and Chen R: LARP1 is regulated by the XIST/miR-374a axis and functions as an oncogene in non-small cell lung carcinoma. Oncol Rep 38: 3659-3667, 2017.

73. Kulkarni P, Dasgupta P, Bhat NS, Shahryari V, Shiina M, Hashimoto Y, Majid S, Deng G, Saini S, Tabatabai ZL, et al: Elevated miR-182-5p associates with renal cancer cell mitotic arrest through diminished MALAT-1 expression. Mol Cancer Res 16: 1750-1760, 2018.

74. Wang QM, Lian GY, Song Y, Huang YF and Gong Y: LncRNA MALAT1 promotes tumorigenesis and immune escape of diffuse large B cell lymphoma by sponging miR-195. Life Sci 231: $116335,2019$.

75. Zhang X, Ma G, Liu J and Zhang Y: MicroRNA-182 promotes proliferation and metastasis by targeting FOXF2 in triple-negative breast cancer. Oncol Lett 14: 4805-4811, 2017. 